\title{
Ion Transport through Diffusion Layer Controlled by Charge Mosaic Membrane
}

\begin{abstract}
Akira Yamauchi
Department of Chemistry, Faculty of Science, Fukuoka University, 8-19-1 Nanakuma, Jonan-ku, Fukuoka 814-0180, Japan

Correspondence should be addressed to Akira Yamauchi, yamakira42@yahoo.co.jp

Received 2 April 2012; Revised 21 June 2012; Accepted 22 July 2012

Academic Editor: Victor V. Nikonenko

Copyright () 2012 Akira Yamauchi. This is an open access article distributed under the Creative Commons Attribution License, which permits unrestricted use, distribution, and reproduction in any medium, provided the original work is properly cited.

The kinetic transport behaviors in near interface of the membranes were studied using commercial anion and cation exchange membrane and charge mosaic membrane. Current-voltage curve gave the limiting current density that indicates the ceiling of conventional flux. From chronopotentiometry above the limiting current density, the transition time was estimated. The thickness of boundary layer was derived with conjunction with the conventional limiting current density and the transition time from steady state flux. On the other hand, the charge mosaic membrane was introduced in order to examine the ion transport on the membrane surface in detail. The concentration profile was discussed by the kinetic transport number with regard to the water dissociation (splitting) on the membrane surface.
\end{abstract}

\section{Introduction}

It is well known that electrodialysis using ion exchange membrane makes drinking water or table salt from sea water. In Japan especially it is popular to use this electrodialysis in various fields such as food, medical pharmacy and ultrapure water [1-3]. However, the more efficient and improved electrodialysis have been required for pure water production because conventional system costs much of electric power at present. One of the main problems originates from the limiting current density that restricts the direct current for supplying through the system and depends on the thickness of diffusion layers. In this study, the way to see the thickness of the depleted solution layer was examined in order to improve the efficiency on the electrodialysis from fundamental standpoint.

As mentioned above, the aim will be focused on the ionic behavior of ion transport from bulk solution to membrane surface. In addition, so far developed charge mosaic membrane [4-6] is introduced into electrodialysis model system and the ion transport mechanism is investigated. As experimental strategies, electrical methods were mainly adopted, and 4 kinds of membrane systems were selected to study: cation exchange membrane system (CMV), anion exchange membrane system (AMV), charge mosaic membrane and cation exchange membrane system (MM + CMV), and anion exchange membrane and charge mosaic membrane system $(\mathrm{AMV}+\mathrm{MM})$.

The potentiometry was also used on the same membrane systems to elucidate the diffusion layer. The chronopotentiometry has been carried out to obtain the transition time [7-9]. The technique was adopted for 4 membrane arrangements to examine the contribution to the boundary layer of the charge mosaic membrane. The kinetic parameter about the boundary layer was noticed and introduced for understanding of the experimental results. The boundary layer thickness was already studied using chronopotentiometric technique in the underlimiting current-range [10], but in this paper different other methods were adopted to measure the boundary layer thickness.

\section{Experimental}

2.1. Materials. The membranes used in the experiments were commercial Selemion CMV as cation exchange membrane, Selemion AMV as anion exchange membrane (Asahi glass Co.), and charge mosaic membrane MM, which was developed in Dainichi Seika. Co. and contained both strong acidtype cation exchange group and strong base-type anion 
exchange group fixed in the direction perpendicular to the membrane surface [4-6]. The $\mathrm{KCl}$ solution was prepared from $10^{-5}$ to $10^{-1} \mathrm{~mol} \mathrm{dm}{ }^{-3}$ in this study.

2.2. Membrane Arrangement. A study in laboratory scale was carried out with 4 kinds of membrane systems, which imagined ED (electrodialysis) and EDI (electrodeionization) model membrane systems.

4 membrane arrangement systems for this research were taken as following.

$\mathrm{CMV}$ : $\mathrm{KCl}$ solution/CMV/KCl solution (ED model).

AMV : $\mathrm{KCl}$ solution/AMV/KCl solution (ED model).

$\mathrm{MM}+\mathrm{CMV}: \mathrm{KCl}$ solution/MM + CMV/KCl solution (EDI model).

$\mathrm{AM}+\mathrm{MMV}: \mathrm{KCl}$ solution/AMV + $\mathrm{MM} / \mathrm{KCl}$ solution (EDI model).

The membranes arrangements of two tops and two bottoms are similar to conventional ED and EDI device, respectively. Then, for convenience, sometimes ED or EDI symbols were used in this paper.

\subsection{Apparatus}

2.3.1. Current-Voltage Curve. Current-voltage curves were determined with a gradual increase of potential difference imposed through the cells, and current density across the membrane was measured. The obtained combination of potential difference and current density gave the experimental current-voltage curve.

2.3.2. Chronopotentiometry. The potentiostat (Potentiostat/Galvanostat, HA-151, Hokutodenko. Co.) allowed the current-voltage measurement and the chronopotentiometry measurement on different membrane arrangements. Chronopotentiogram was driven with a function generator (function generator, HB-111, Hokutodenko. Co.) and recorded by X-Y-t recorder (XY recorder F-35C, Riken Denshi. Co.). The experimental cell for the current-voltage measurement and the chronopotentiometry was the fourelectrodes cell as depicted in Figure 1. The voltage drops across the membranes under investigation are measured by Haber-Luggin capillaries in which $3 \mathrm{M} \mathrm{KCl}$ solution was filled with agar and each connected to potentiostat or galvanostat where $\mathrm{Ag} / \mathrm{AgCl}$ electrode was connected as reference electrode.

Chronopotentiometry is one of the electrochemical characterization methods that measure the electric potential response of a system to the imposed current [10-12]. It was performed with 4 membrane arrangements under investigation. It is not the same measurement as the current-voltage curve measurement at the point where the current instead of voltage drop was applied into the system by means of outer supply. The method of chronopotentiometry was firstly developed for electrochemistry of electrode and now used also for studies in membrane science field almost without any changes [13]. Then the Sand equation, which had concerned with electrode reaction was introduced toward the membrane system.

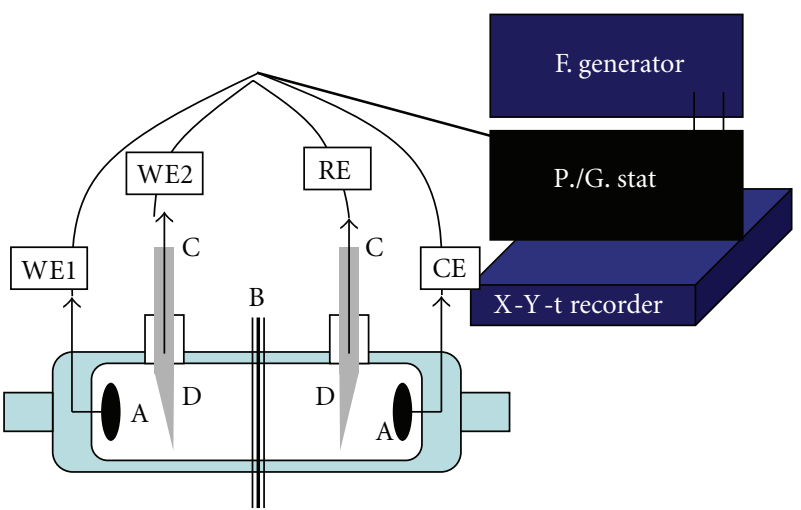

Figure 1: Experimental cell with electrochemical apparatus. A; $\mathrm{Ag} / \mathrm{AgCl}$ electrode. B: Membrane system (Monopolar or Monopolar + Mosaic membranes). Membranes parts are fixed by two rubbers with holes, $1 \mathrm{~cm}$ in diameter from both sides to avoid the leak of solution. $\mathrm{C}$ : $\mathrm{Ag} / \mathrm{AgCl}$ electrode. $\mathrm{D}: 3 \mathrm{M} \mathrm{KCl}$ in agar.

The transition time obtained by chronopotentiometry corresponds to the moment, where the initial salt concentration on the membrane surface decrease until it becomes zero [12]. On the other hand, it means the time to reach overlimiting current density by applied current. The obtained kinetic parameters were analyzed by the Sand equation.

2.3.3. $\mathrm{pH}$ Measurement. $\mathrm{pH}$ indicators, phenolphthalein, and methyl red were put into the $\mathrm{KCl}$ solution to check simply the occurrence of water dissociation during operation of chronopotentiometry. It indicated that the $\mathrm{pH}$ change, $\mathrm{H}^{+}$ take place from slight red color qualitatively.

\section{Results and Discussion}

3.1. Limiting Current Density. Current-voltage curve measurement was carried out to obtain the limiting current density in the membrane systems of 4 arrangements under investigation. The limiting current density was determined from current-voltage curve and explained by the well-known schematic picture [11] in which three regions can be distinguished. Firstly, a linear part appears in the low-potential drop region, called the ohmic region. Secondly, the current density attains a plateau region due to the depleted ion layer adjacent to membrane surface. The concentration on the membrane surface became zero finally. Hence the current density on this step was defined as the limiting current density, and there the salt flux is dominated by the diffusion between the bulk solution and membrane surface. After the plateau, the current density begins to increase again. The third region is called the overlimiting region [11]. The limiting current densities about ED or EDI model system were determined from the current-voltage curves. In fact, the limiting current density on EDI was slightly larger than on ED. The salt flux across the membrane is in agreement with the salt flux, which passed from the bulk solution to the membrane surface. Therefore, it is important to consider the flux until the concentration on the membrane surface reach zero. 


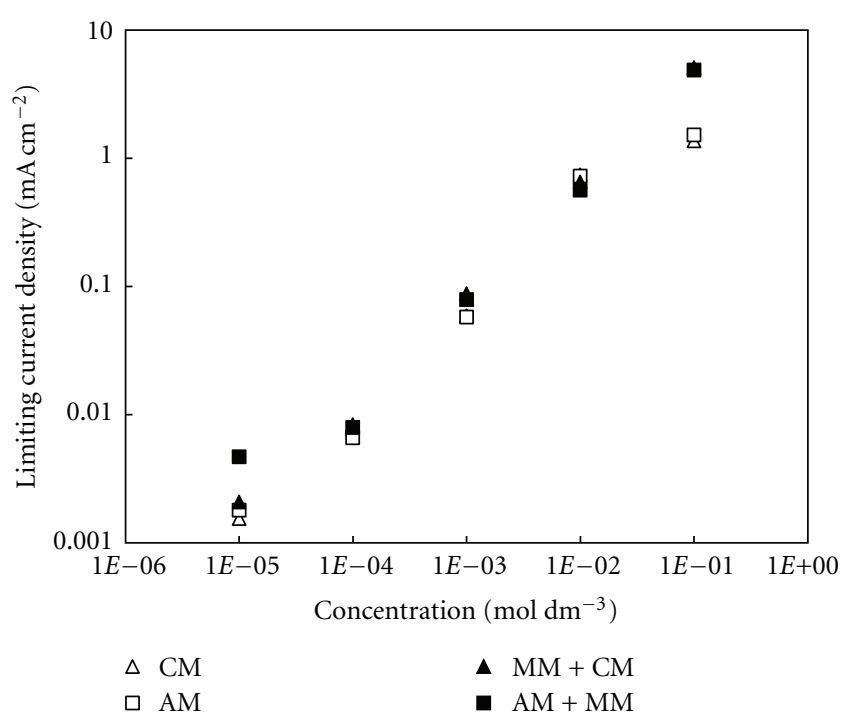

FIGURE 2: Limiting current density of 4 membrane arrangements in $\mathrm{KCl}$ solution. Symbols are inserted in the figure.

The limiting current density is given by a following equation derived in the literatures [10-14]:

$$
i_{\lim }=\frac{F D_{\mathrm{KCl}} C_{O}}{\delta\left(\bar{t}_{i}-t_{i}\right)} .
$$

Here $F$ is Faraday constant, $C_{O}$ the bulk solution concentration, $D$ the salt diffusion coefficient, $\delta$ the diffusion boundary thickness, $\overline{t_{i}}$ the counter ion transport number in the membrane, and $t_{i}$ the transport number in the solution.

Here if one assumes that $D$ the salt diffusion coefficient, $\delta$ the diffusion boundary thickness and $\overline{t_{i}}$ the counter ion transport number in the membrane are known, $t_{i}$ the transport number in the solution can be obtained. It will be done about the detailed discussion later.

3.2. Transition Time. Chronopotentiogram, one of electrochemical methods, is given as schematic explanation in Figure 3. The result in $0.01 \mathrm{M} \mathrm{KCl}$ solution with using CMV was shown in Figure 4. All current densities imposed for the chronopotentiometry were always larger than the limiting current density obtained by the current-voltage curve measurement. In the case where such a large current was imposed on the membrane system, the concentration polarization should occur rapidly on the membrane surface and the concentration on the membrane surface becomes zero immediately. The situation is the same as the case mentioned in previous section. The transition time, $\tau$ was defined as the time when the concentration on the membrane surface became zero, that is, inflection point of shadow in Figure 3.

Now, the transition time is given by Sand as following equation $[12,13]$ :

$$
\tau=\frac{\pi D_{\mathrm{KCl}}}{4} \frac{1}{i^{2}}\left(\frac{F z_{i} C_{O}}{\overline{t_{i}}-t_{i}}\right)^{2}
$$

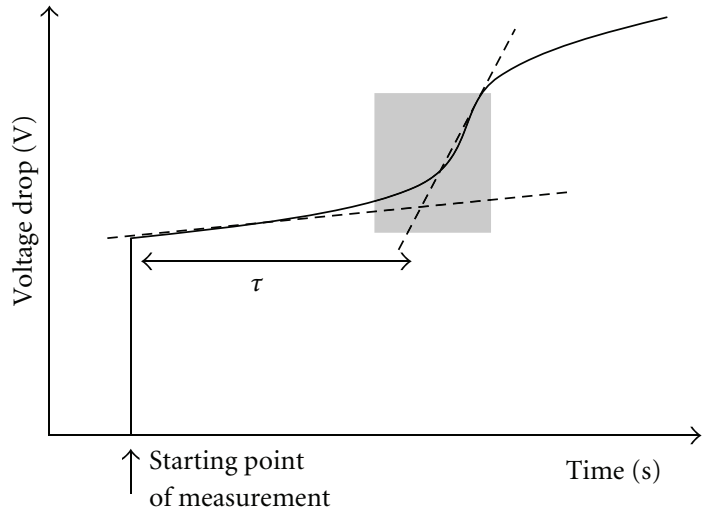

FIGURE 3: Schematic drawing of chronopotentiogram.

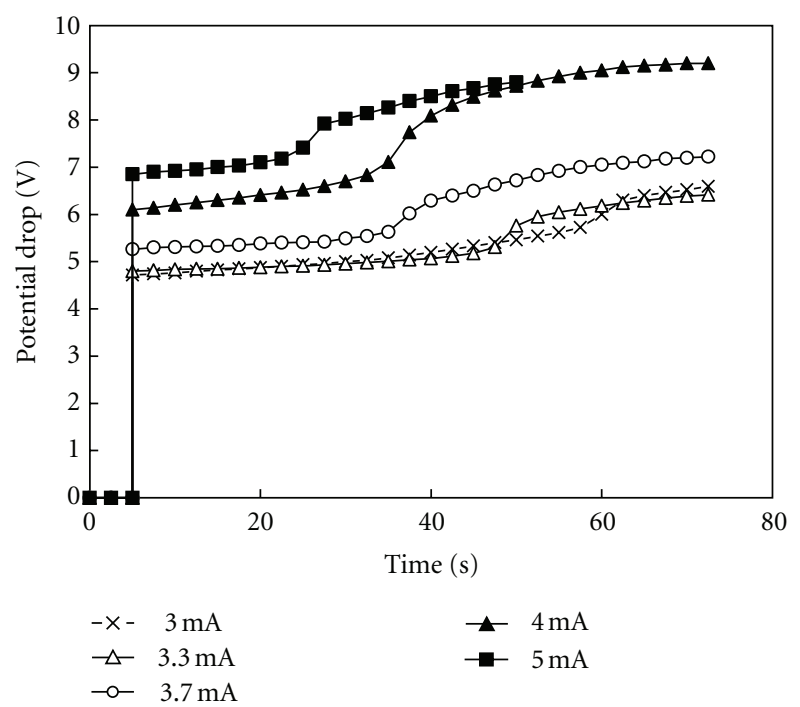

FIgURE 4: Chronopotentiogram with a cation exchange membrane in $0.01 \mathrm{M} \mathrm{KCl}$ solution. Current densities are inserted in the figure.

Here $D$ is the diffusion coefficient of electrolyte, $C_{O}$ and $z_{i}$ the concentration in the bulk and the charge of the counter ion, $\overline{t_{i}}$ and $t_{i}$ the transport number of the counter ion in the membrane and solution, respectively, $i$ the current density, and $F$ the Faraday coefficient. Then the measured transition times were indicated as a function of inverse of square of current densities in Figure 5. According to Figure 5, there were hardly difference between CMV and AMV membrane arrangements. On the other hand, in the case where the charged mosaic membrane was introduced additionally on cation- and anion exchange membrane like arrangements, $\mathrm{MM}+\mathrm{CMV}$ and $\mathrm{AMV}+\mathrm{MM}$, the transition time was changed remarkably. The transition time, $\tau$, increased on the arrangement, $\mathrm{MM}+\mathrm{CMV}$, while $\tau$ on the arrangement, AMV + MM decreased. At present, it is not clear, but there seems to be influence of boundary layer, $\delta$.

3.3. Kinetic Transport Number in Boundary Layer. When $z_{i}=$ 1 , (2) was rearranged to evaluate the transport number at 


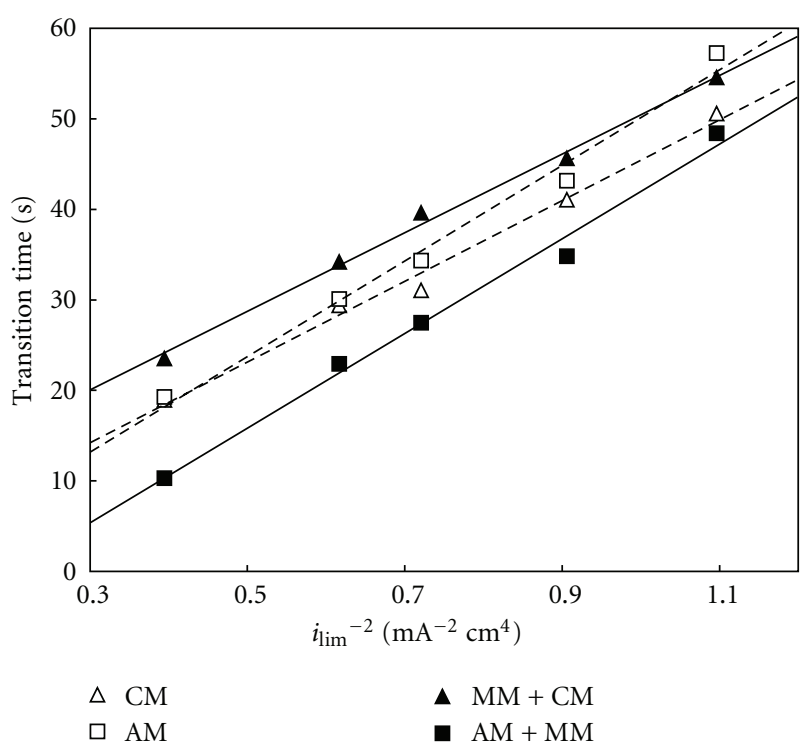

FIgURe 5: Transition time versus $i_{\text {lim }}{ }^{-2}$ in $0.01 \mathrm{M} \mathrm{KCl}$ solution. Symbols are inserted in the figure.

the boundary layer in the case of $\mathrm{KCl}$ solution as follows:

$$
t_{i}=\bar{t}_{i}-C_{O} F \frac{1}{i} \sqrt{\frac{\pi D_{\mathrm{KCl}}}{4 \tau}} .
$$

Potential changes along the time and increases after the current density value reached on the transition time are shown at the grey portion in Figure 3. The fact suggests that any ion transport process also changes after the transition time. The transport number might be changed under a certain condition, although the transport number had been almost unity in membrane when the relation between the voltage and the current has been simply ohmic at the moment. The kinetic transport number on the membrane surface was defined and derived from the above (3) on the moment when ion concentrations on the membrane reached zero.

The kinetic transport number in the boundary layer was shown in Figure 6, where the diffusion coefficient was assumed to be constant values in $\mathrm{KCl}$ solution and also transport number in membrane, $\overline{t_{i}}=1$. According to Figure 6, an addition of the charged mosaic membrane resulted in the change of transport number at the boundary layer and the transport number tended to change remarkably with the extent of current density. In particular, it was supposed that the kinetic transport number in the boundary layer decreased remarkably in the case of AM + MM. Hence we define the transport number as kinetic transport number in boundary layer.

3.4. Thickness of Boundary Layer. Equation (1) on the limiting current density was rearranged to derive the thickness of boundary layer as follows:

$$
\delta=\frac{F D_{\mathrm{KCl}} C_{O}}{i_{\lim }\left(\bar{t}_{i}-t_{i}\right)} .
$$

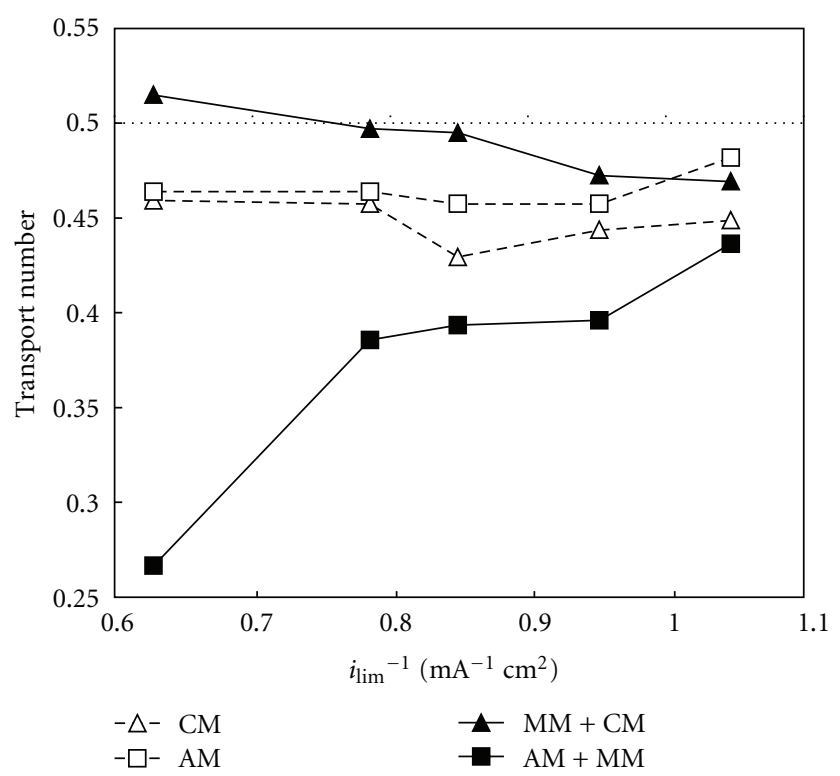

FIGURE 6: Relation between transport number and various $i_{\lim }{ }^{-1}$ value in $0.01 \mathrm{M} \mathrm{KCl}$ solution on 4 membrane arrangements. Symbols are inserted in the figure.

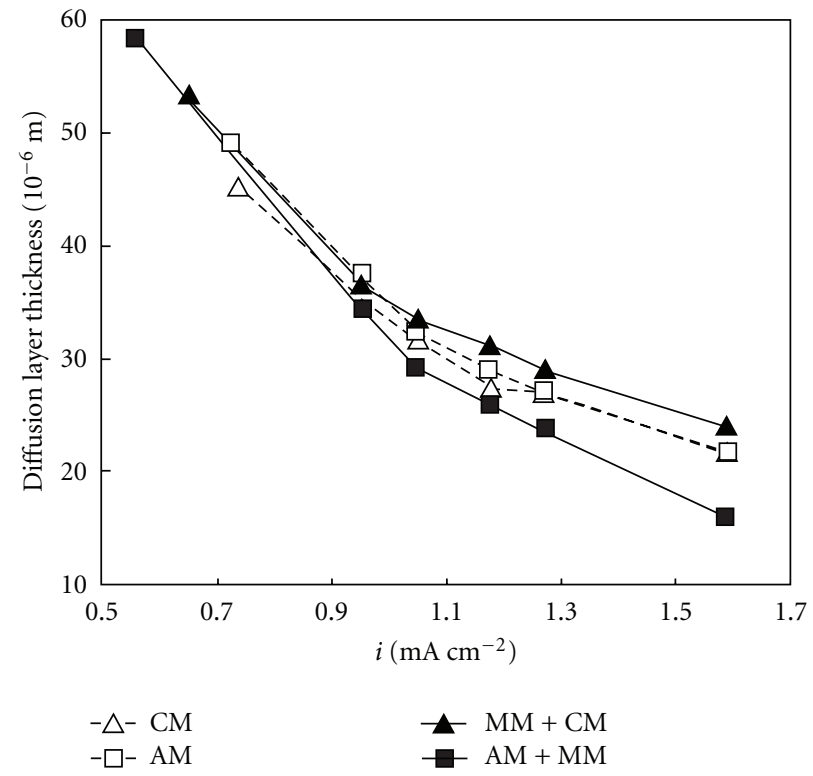

Figure 7: Diffusion layer thickness versus current density. Symbols are inserted in the figure.

The estimated limiting current density was already given in Figure 2. The transport number on designated ion should be unity through the membrane. This condition was equivalent with that in the chronopotentiometry at the transition time just before. It was supposed that the transport number and the current density obtained from the chronopotentiometry were able to be substituted by using (4) because the conditions on the surface of membrane are same. Then, the thickness of the boundary layer was represented in Figure 7. The added charge mosaic membrane 


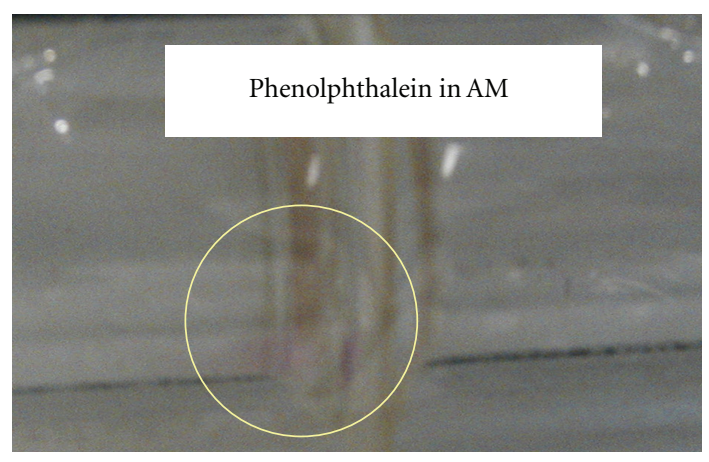

(a)

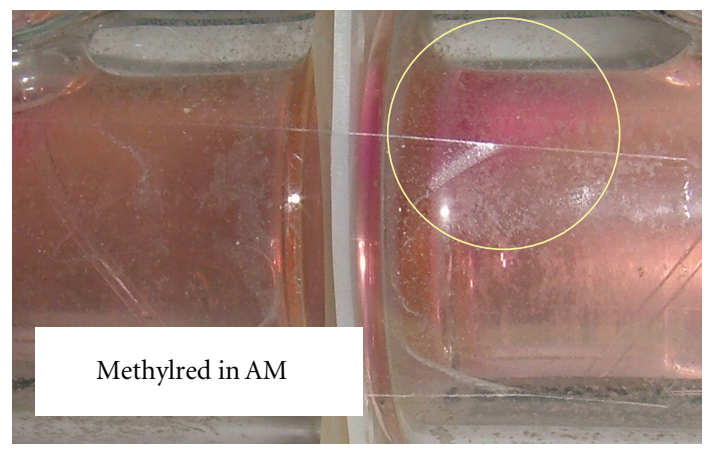

(c)

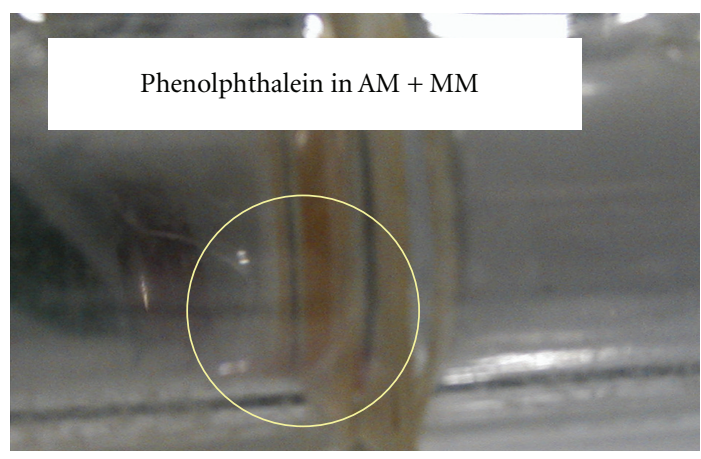

(b)

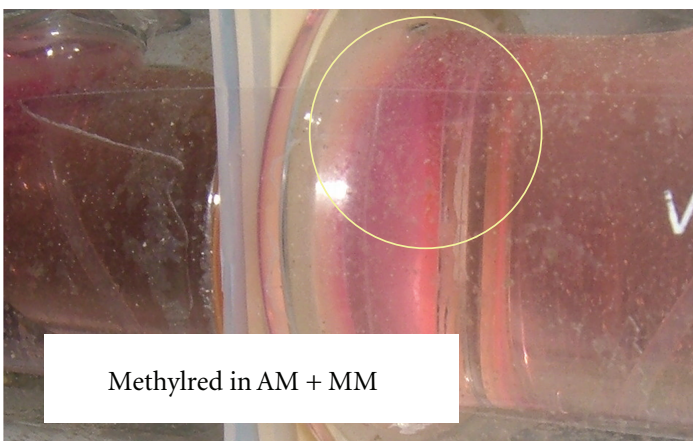

(d)

FIGURE 8: Indicator, methyl red in cell of two bottoms changed in red. This shows the existence of $\mathrm{H}^{+}$in a bulk solution.
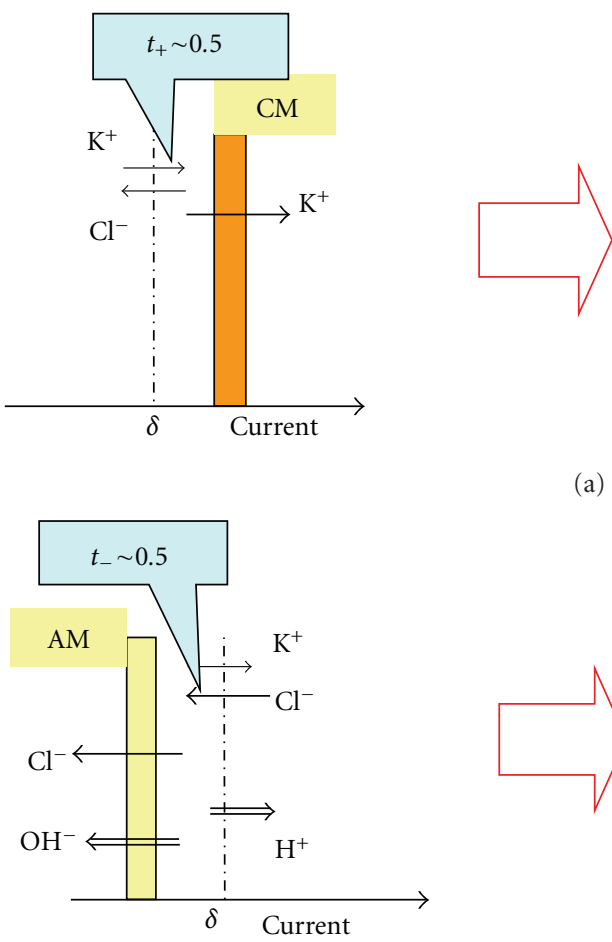

(a)
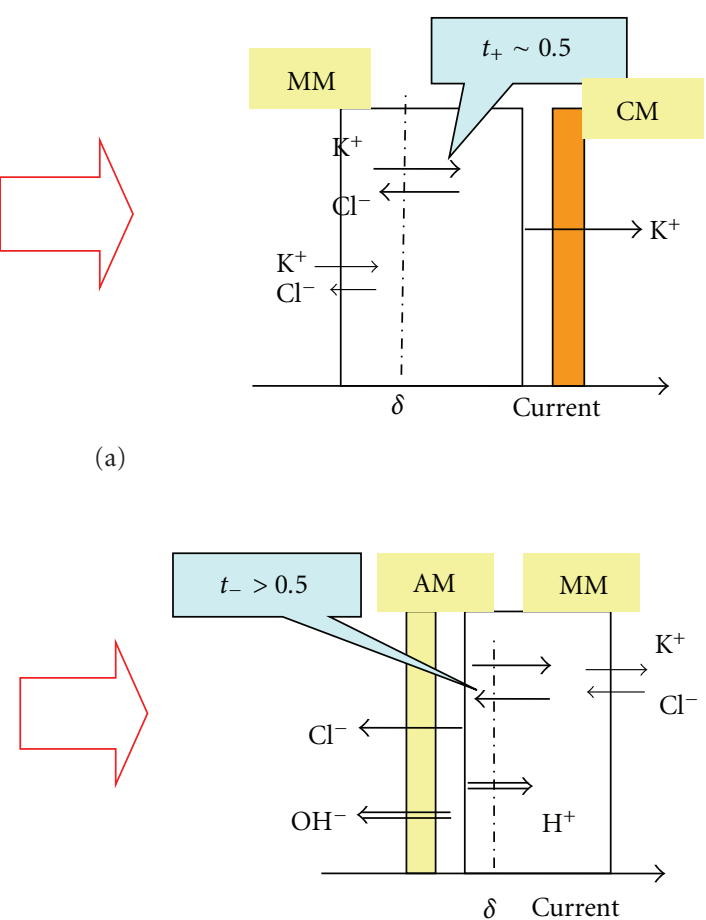

(b)

Figure 9: Schematic model of ion transport on the membrane surface. Dotted lines are hypothetical boundary lines. 
caused the increase of the thickness of boundary layer on the cation exchange membrane and the decrease on the anion exchange membrane. The tendency of change became remarkable depending on the current density.

Finally, it was suggested that the introduction of the charge mosaic membrane into ion exchange membrane resulted in the change of boundary layer's thickness, and this result supported the speculation in Section 3.2. Transition Time.

3.5. Water Splitting. During the chronopotentiometry, the $\mathrm{pH}$ measurement was carried out at the same time under the presence of $\mathrm{pH}$ indicators, phenolphthalein, and methyl red, and the results were shown in Figure 8. The color changes were not recognized in CMV and MM + CM systems at all, but the red color changes appeared in AMV and $\mathrm{AM}+\mathrm{MM}$ systems. The $\mathrm{pH}$ change suggested that the water splitting took place near the surfaces on anion exchange membrane systems only. The water splitting seems to give an affect to the ion transport process on membrane, in particular anion membrane systems. Then the experimental results were tentatively examined by the following model in Figure 9.

The arrow toward right indicates the current direction in Figure 9 on which the dotted line is hypothetically located as the end of diffusion layer from each membrane surface. Cation passage through the cation exchange membrane is $\mathrm{K}^{+}$in the Figure $9(\mathrm{a})$ (left) because the transport number on the cation exchange membrane is theoretically unity. $\mathrm{K}^{+}$is also transported toward the current direction in the diffusion layer and $\mathrm{Cl}^{-}$is transported toward the reverse direction. The width of arrow depends on the amount of transported ion in Figure 9.

The arrangement, $\mathrm{MM}+\mathrm{CMV}$, is represented in Figure 9(a)(right) where the water splitting phenomenon on the membrane did not appear but the diffusion layer indicated the growth by introduction of the charge mosaic membrane on the cation exchange membrane as indicated in Figure 7. This is caused by that ion transport process may depend on difference in movement of ions in a mosaic membrane and in solution under overlimiting current. In other words, the ions in free solution, cation, and anion affected each other by the interaction between the ions of the positive and negative to some extent, while ions in charge mosaic membrane under electricity moves smoothly along the fixed charge sites faster than them in free solution. It means the increase of $\mathrm{K}^{+}$transport number and finally the thickness of diffusion layer will be reflected from the equivalent properties of (3) and (4) at the same limiting current.

The arrangement, AMV, was shown in Figure 9(b)(left) on which the water splitting was observed sufficiently. In the case, the produced $\mathrm{H}^{+}$seems to affect the $\mathrm{Cl}^{-}$transport number. The transport numbers, however, did not change so much as seen in Figure 6 because the protons would be compensated by $\mathrm{Cl}^{-}$of high concentration in bulk solution next to the diffusion layer.

The arrangement, AMV $+\mathrm{MM}$, was indicated in Figure 9 (b)(right). The transport number was remarkably changed on this model, as clear from the result in Figure 6, and the diffusion layer turned to be decreased as shown in Figure 7. It is supposed that the water splitting occurred at the membrane surface participates in the decrease. The produced protons promptly are connected with $\mathrm{Cl}^{-}$on mosaic membrane introduced to this systems. As one possibility, though the situation is not clear, it is guessed as follows. As the $\mathrm{Cl}^{-}$from the bulk solution is slow rate compared to those in mosaic membrane, $\mathrm{Cl}^{-}$could not compensate the anionic depletion due to the produced proton in the diffusion layer. As a result, the anion transport number would decrease or the thickness of diffusion layer would be getting smaller. Figure 8 indicated the color's change by methyl red indicators. The red sign due to a generated proton appeared on the solution toward the cathode. It means that $\mathrm{H}^{+}$was transported toward the bulk solution as a part of charge carriers from the end of diffusion layer.

According to Figure 9, it was supposed that the ionic transport was changed by an addition of the charge mosaic membrane to electrodialysis.

\section{Conclusion}

Kinetic transport number on ionic transport in the diffusion layer in near the membrane surface was obtained by means of the chronopotentiometry. The equation was developed to obtain the thickness of the diffusion layer from equation of limiting current density. The diffusion layers were indicated as function given current densities.

On the other hand, the water splitting phenomenon was observed from $\mathrm{pH}$ measurement in both cases of anion exchange membrane system and anion exchange + charge mosaic membrane system. The phenomena together with relation to the thickness of diffusion layer were considered schematically in Figure 9.

In this study, it was examined whether the limit of current density could be increased by introducing the charge mosaic membrane.

\section{References}

[1] T. Sata, "Ion exchange membrane," in Royal Society of Chemistry, pp. 215-240, London, UK, 2004.

[2] Y. Tanaka, Ion Exchange Membrane: Fundamentals and Applications, vol. 12 of Membrane Science and Technology, Elsevier, Amsterdam, The Netherlands, 2007.

[3] T. Hamano, P. B. Chebi, L. Gerdes, and T. D. Wolfe, "The development of a new electrodialysis system for brackish water," Desalination, vol. 96, pp. 23-31, 1994.

[4] A. Yamauchi, J. Tateyama, B. I. Etoh, M. Takizawa, Y. Sugito, and S. Doi, "Charged mosaic membrane prepared from microsphere gel and its characterization," Journal of Membrane Science, vol. 173, no. 2, pp. 275-280, 2000.

[5] M. Takizawa, Y. Sugito, N. Oguma, M. Nakamura, S. Horiguchi, and T. Fukutomi, "Charge-mosaic membrane prepared from microspheres," Journal of Polymer Science A, vol. 41, no. 9, pp. 1251-1261, 2003.

[6] D. L. Grzenia, A. Yamauchi, and S. R. Wickramasinghe, "Electrolyte dialysis using charge-mosaic membranes," Desalination and Water Treatment, vol. 4, pp. 306-310, 2009. 
[7] N. Pismenskaia, P. Sistat, P. Huguet, V. Nikonenko, and G. Pourcelly, "Chronopotentiometry applied to the study of ion transfer through anion exchange membranes," Journal of Membrane Science, vol. 228, no. 1, pp. 65-76, 2004.

[8] J. J. Krol, M. Wessling, and H. Strathmann, "Concentration polarization with monopolar ion exchange membranes: current-voltage curves and water dissociation," Journal of Membrane Science, vol. 162, no. 1-2, pp. 145-154, 1999.

[9] J. J. Krol, M. Wessling, and H. Strathmann, "Chronopotentiometry and overlimiting ion transport through monopolar ion exchange membranes," Journal of Membrane Science, vol. 162, no. 1-2, pp. 155-164, 1999.

[10] P. Sistat and G. Pourcelly, "Chronopotentiometric response of an ion-exchange membrane in the underlimiting currentrange. Transport phenomena within the diffusion layers," Journal of Membrane Science, vol. 123, no. 1, pp. 121-131, 1997.

[11] R. Ibanez, D. F. Stamatialis, and M. Wessling, "Role of membrane surface in concentration polarization at cation exchange membranes," Journal of Membrane Science, vol. 239, no. 1, pp. 119-128, 2004.

[12] A. J. Bard and L. R. Faulkner, Electrochemical Methods, Fundamentals and Applications, Wiley International Edition, 1980.

[13] A. Yamauchi, A. M. E. Sayed, K. Mizuguchi, M. Kodama, and Y. Sugito, "Ion transport behavior in diffusion layer of new designed ion exchange-mosaic composite polymer membrane," Journal of Membrane Science, vol. 283, no. 1-2, pp. 301-309, 2006.

[14] T. Yawataya, Ion Exchange Membrane For Engineer, Kyoritsu Shuppan, 1982. 

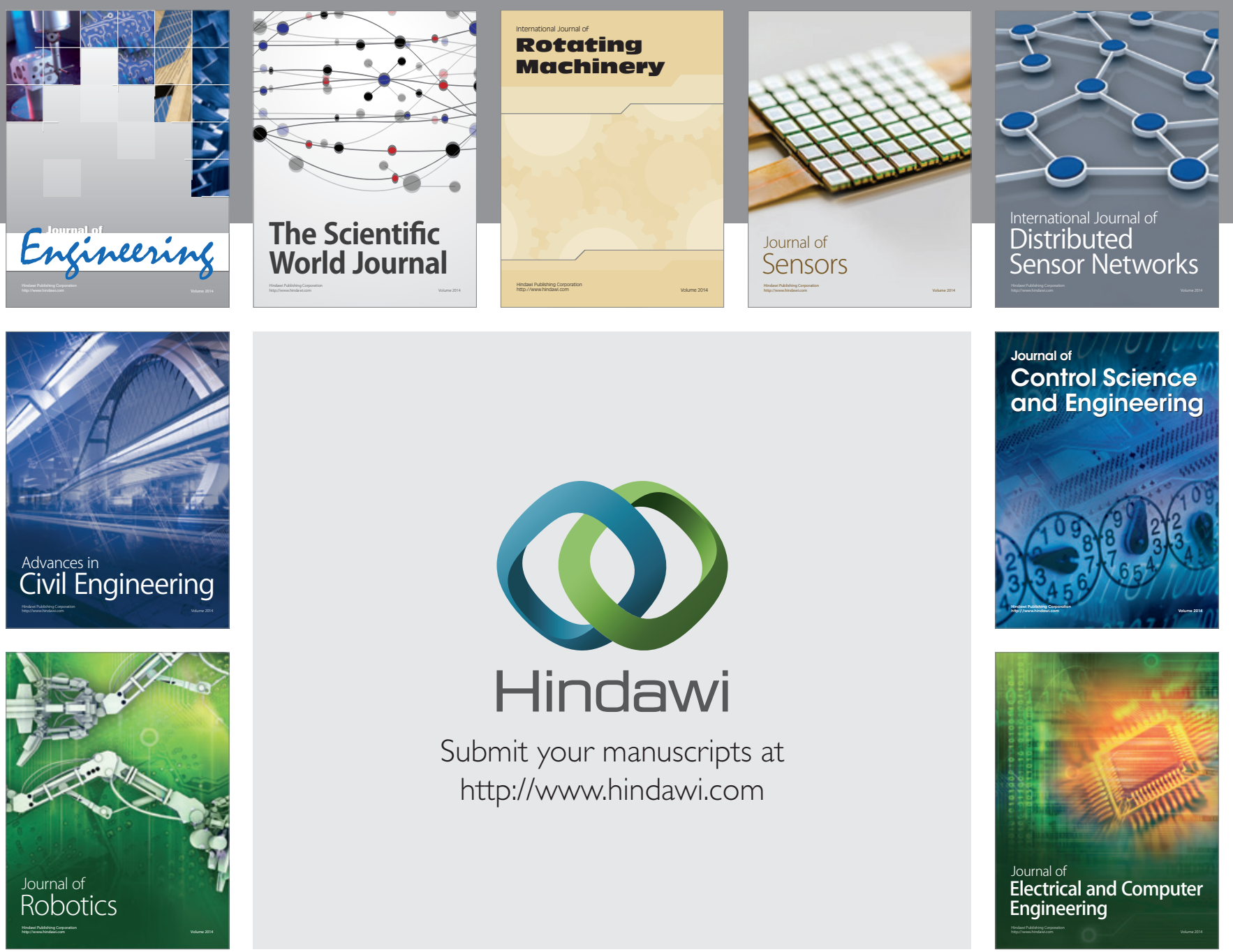

Submit your manuscripts at

http://www.hindawi.com
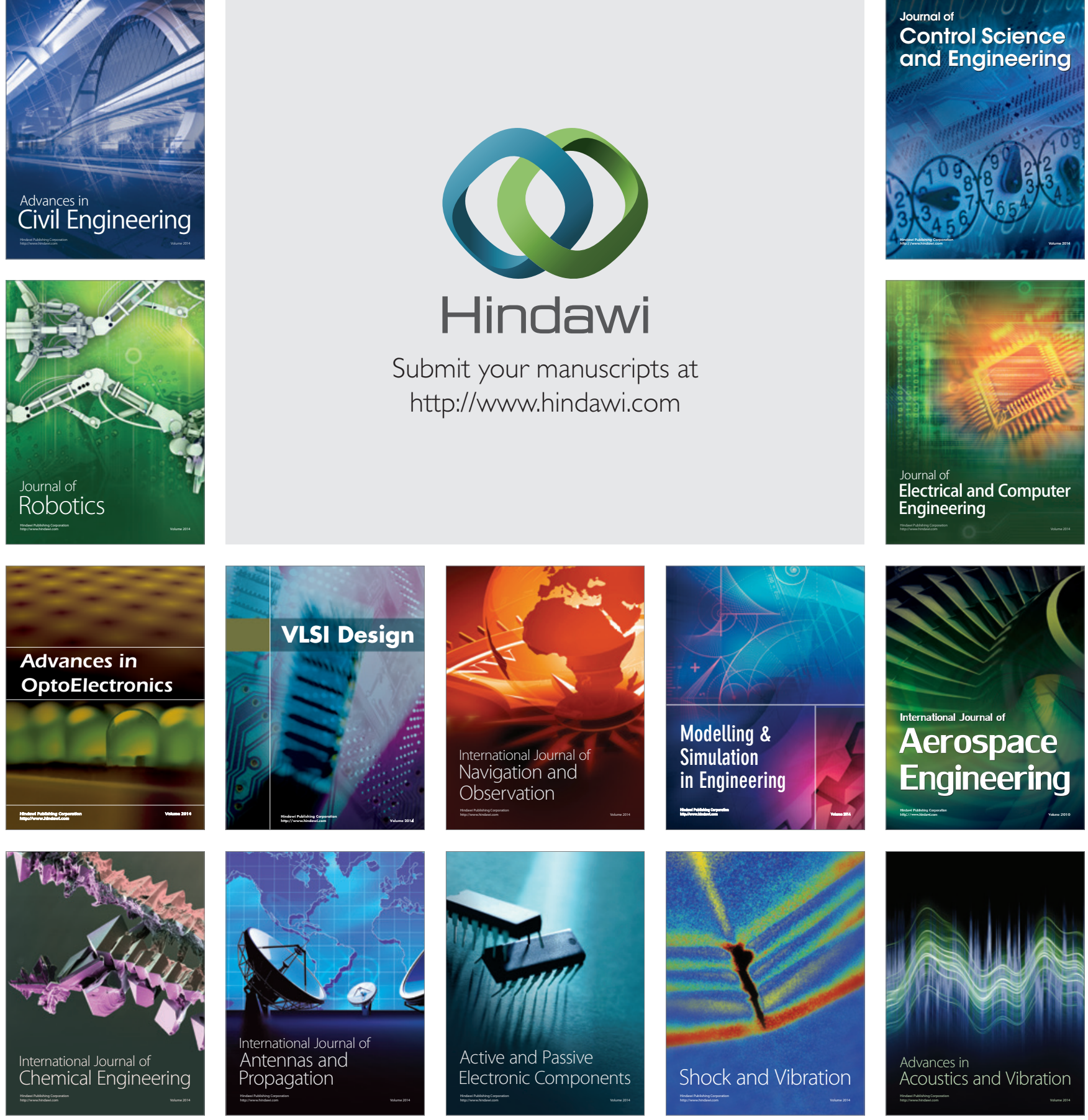